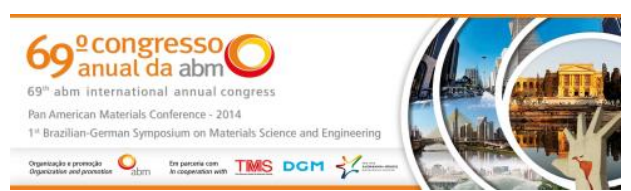

Tema: Produtos metálicos não-ferrosos

\title{
EFEITO DE IMPUREZAS NAS PROPRIEDADES MECÂNICAS DO COBRE UNS C12200*
}

\author{
Victor Caso Moreira ${ }^{1}$ \\ André Paulo Tschiptschin²
}

\section{Resumo}

As propriedades mecânicas de tubos de cobre ligado ao fósforo (liga UNS C12200 cobre DHP) são importantes para sua aplicação nos produtos finais, em que processos de expansão e dobra são fundamentais. Três grupos de amostras obtidas por extrusão e trefilação (deformação a frio de $80 \%$ ) e com diferentes níveis de impurezas (184 ppm a $319 \mathrm{ppm}$ ) foram submetidos a tratamento de recozimento e tiveram suas propriedades mecânicas e microestrutura analisadas. Os resultados obtidos nesse trabalho mostram que as propriedades do material variam com o teor de impurezas, devido aos mecanismos de endurecimento por solução sólida e por redução de tamanho de grão. Os resultados são mais pronunciados sobre o limite de escoamento e de resistência à tração. Em termos de alongamento, observa-se que a homogeneidade microestrutural é determinante e tem relação com o teor de impurezas, possivelmente devido à ocorrência de recristalização secundária por flutuações de composição química. $O$ aumento do teor de impurezas parece aumentar a temperatura de recristalização do material, diminuindo o tamanho de grão resultante do recozimento, o que confirma os dados da literatura para o cobre eletrolítico.

Palavras-chave: Cobre DHP; C12200; Recozimento; Recristalização.

\section{EFFECT OF IMPURITIES ON MECHANICAL PROPERTIES OF COPPER UNS} C12200

\section{Abstract}

The mechanical properties of deoxidized high phosphorus copper tubes (UNS C12200 alloy - DHP copper) are an important issue to its application, which demands expansion and bending processes. Three group of samples hot extruded and drawn $(80 \%$ of cold working) and with different purities were annealed. The mechanical properties and microstructures of the samples were analyzed after the heat treatment. The results show a variation of the properties of the material with the impurities content by means of solid solution and grain boundary strengthening mechanisms. The variation is stronger when yield strength and tensile strength are analyzed. The microstructure homogeneity is more determinative to elongation results and it has some relation with the impurities content, due probably to secondary recrystallization owing to chemical composition fluctuations. Impurities seem to rise the recrystallization temperature of the alloy, providing smaller grain size after annealing, which is in accordance with the literature for electrolyte copper.

Keywords: DHP copper; C12200; Annealing; Recrystallization.

1 Engenheiro Metalurgista, PMT, EPUSP, Paranapanema AS, Santo André, SP, Brasil.
2 Professor Titular, PMT, EPUSP, São Paulo, SP, Brasil.

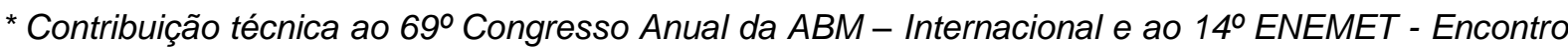
Nacional de Estudantes de Engenharia Metalúrgica, de Materiais e de Minas, 21 a 25 de julho de 2014, São Paulo, SP, Brasil.
} 


\section{INTRODUÇÃO}

Tubos de cobre ligado ao fósforo (liga UNS C12200) são largamente usados em equipamentos de refrigeração e na construção civil para o transporte de gás e águas fria e quente. Condutividade térmica e resistência à corrosão em meio aquoso são as características mais relevantes na seleção desse material. No entanto, esses produtos serão ainda conformados mecanicamente na montagem dos produtos finais - nomeadamente, processos de expansão e dobra -, tonando importantes as propriedades mecânicas do material, em especial o alongamento e a redução de área. O fósforo é adicionado ao cobre visando à desoxidação do metal líquido, à estabilidade do material em operações de soldagem e à homogeneidade microestrutural que resultará em alongamento e redução de área necessários à aplicação, sem perda significativa da resistência à corrosão e da condutividade térmica.

Alguns trabalhos foram publicados relacionando a concentração de impurezas com as propriedades mecânicas de vergalhões de cobre eletrolítico. Chmielarz e outros [1] correlacionaram estatisticamente as propriedades mecânicas medidas por AR Test com a composição química de diversos vergalhões, encontrando a seguinte ordem de influência (negativa) dos elementos no alongamento do material: $\mathrm{Sn}>\mathrm{Sb}$ $>\mathrm{As}>\mathrm{Pb}>\mathrm{Ni}>\mathrm{Fe}>\mathrm{Zn}>\mathrm{S}>\mathrm{Ag}$. Silva e outros [2] demonstraram que a variação no resultado de ARTest está relacionada à mudança na temperatura de recristalização do cobre eletrolítico, devido às impurezas presentes no material. Os autores chamam atenção para a importância de um teor adequado de oxigênio, capaz de precipitar as impurezas e diminuir a temperatura de recristalização, além de citarem o efeito combinado que alguns elementos apresentam no cobre (como Pb e S, que em conjunto podem se precipitar e não influenciar a recristalização). Devese ressaltar, no entanto, que os resultados dizem respeito ao cobre eletrolítico, no qual os teores de impurezas são muito menores que os encontrados no cobre fosforoso.

Em termos de recristalização, Pitt e outros [3] demonstraram que algumas dezenas de ppm de Sn, Se e Ag são capazes de causar aumentos de dezenas de graus Celsius na temperatura de recristalização do cobre eletrolítico. Jakani e outros [4], também estudando cobre eletrolítico, determinaram a importância do teor de enxofre para a evolução do processo de recristalização. Nesse estudo, os autores apontam para o aumento de $2 \mathrm{ppm}$ para $8 \mathrm{ppm}$ no teor de enxofre como provável causa do aumento da temperatura de recristalização e da mudança na textura final após o tratamento térmico, o que pode ser considerado em discrepância com os resultados apresentados por Chmielarz e colaboradores [1]. Segundo os autores, o enxofre, devido à interação com discordâncias, parece retardar o desenvolvimento de núcleos de recristalização, além de restringir a movimentação de contornos de grão, provavelmente por meio de segregação inter-granular. Coutsouradis e colaboradores [5] também encontraram forte influência do teor de enxofre e outros elementos, como $\mathrm{Pb}$ e $\mathrm{Bi}$, na temperatura de recristalização do cobre.

O objetivo deste trabalho é a avaliação da influência do teor de impurezas na microestrutura e nas propriedades mecânicas de tubos de cobre fosforoso, devido à importância dessas propriedades na aplicação do produto. Os resultados serão comparados com os dados da literatura para o cobre eletrolítico, uma vez que não foram encontrados resultados para cobre fosforoso.

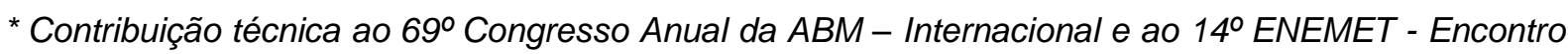
Nacional de Estudantes de Engenharia Metalúrgica, de Materiais e de Minas, 21 a 25 de julho de 2014, São Paulo, SP, Brasil.
} 


\section{MATERIAIS E MÉTODOS}

Três grupos de amostras de tubos de cobre liga C12200 (cobre DHP - deoxidized high phosphorus), obtidos por extrusão e trefilação (deformação a frio de $80 \%$ ) e com diferentes níveis de pureza (Tabela 1), foram submetidos simultaneamente a tratamento de recozimento. A influência do teor de pureza da liga foi avaliada por meio de ensaios de tração uniaxial e análise metalográfica. As quantidades de fósforo e oxigênio das amostras são semelhantes, e as principais impurezas encontradas são: $\mathrm{Sn}, \mathrm{Ni}$, $\mathrm{Fe}$ e Zn.

Tabela 1. Composição química dos 3 grupos de amostras de tubos de cobre C12200

\begin{tabular}{ccc}
\hline \multirow{2}{*}{ Grupo } & \multicolumn{2}{c}{ Composição, \% } \\
& $\mathrm{Cu}+\mathrm{P}+\mathrm{O}$ & $\sum$ Impurezas \\
\hline 1 & 99,98 & 0,0184 \\
2 & 99,97 & 0,0277 \\
3 & 99,97 & 0,0319 \\
\hline
\end{tabular}

Os corpos de prova dos ensaios de tração foram fresados nas dimensões apresentadas na Figura 1 e tracionados na direção correspondente à longitudinal dos tubos. A máquina de tração utilizada foi uma Tinius Olsen e a velocidade de afastamento das garras foi de $30 \mathrm{~mm} / \mathrm{min}$. A força aplicada pelo equipamento e 0 deslocamento das garras foram coletados por computador conectado à máquina e convertidos para tensão e deformação de engenharia; para a primeira foi utilizada uma fórmula que leva em consideração o raio de curvatura e espessura do tubo de origem, enquanto para a segunda o cálculo foi feito tendo como base o comprimento inicial de $50,8 \mathrm{~mm}$. Tanto as dimensões do corpo de prova como a fórmula utilizada no cálculo de tensão de engenharia atendem à norma ASTM E8.

A análise metalográfica foi realizada na direção longitudinal dos tubos (Figura 1) e o tamanho de grão foi analisado pelo método comparativo. As amostras foram preparadas por lixamento, polimento e ataque químico em ácido nítrico. A microestrutura foi observada com auxílio de um microscópio Philips modelo XL30.

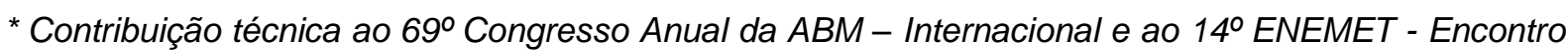
Nacional de Estudantes de Engenharia Metalúrgica, de Materiais e de Minas, 21 a 25 de julho de 2014, São Paulo, SP, Brasil.
} 

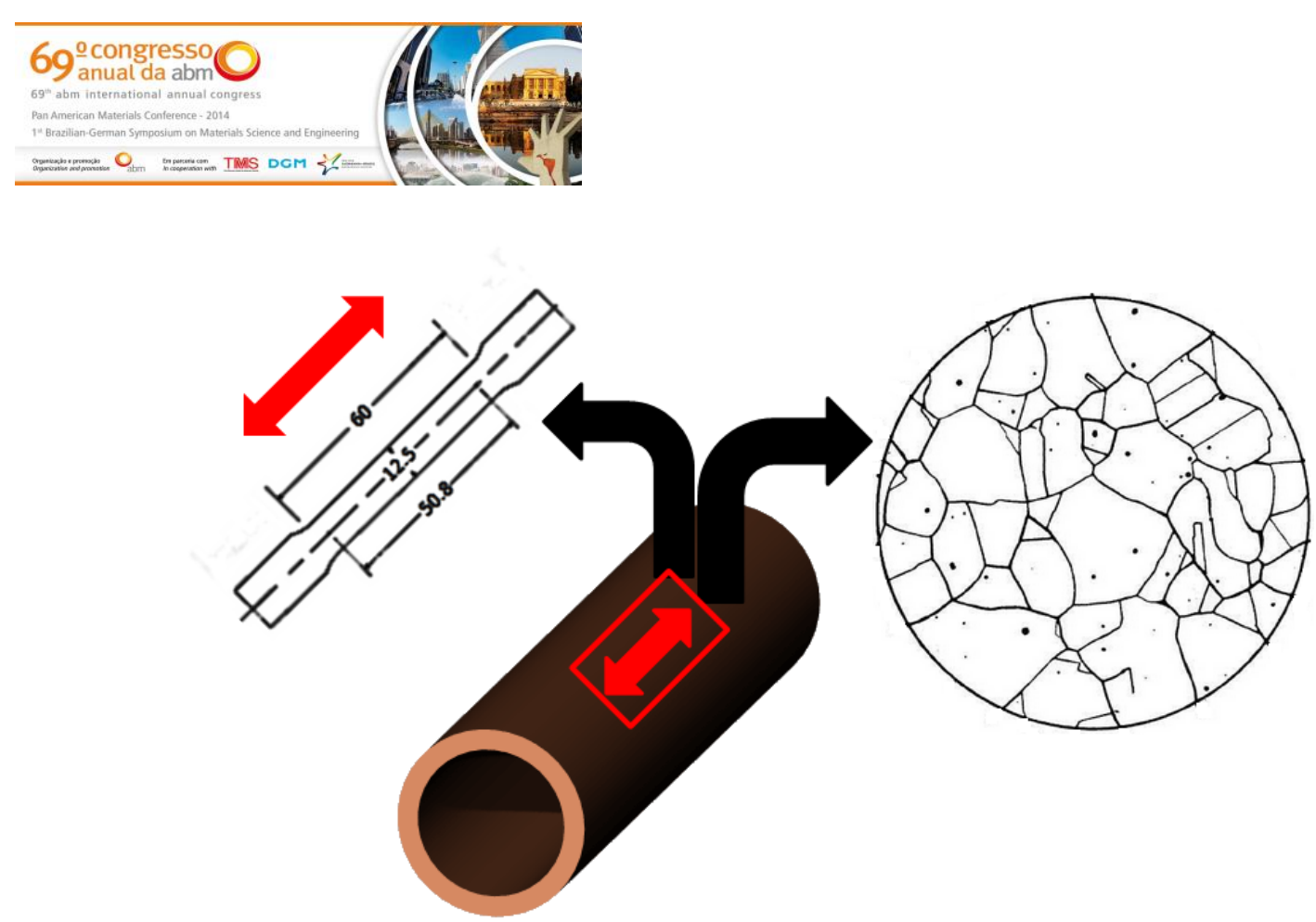

Figura 1. Direção de realização dos ensaios e geometria dos corpos de prova de tração

\section{RESULTADOS}

Como se observa na Tabela 2, o comportamento das amostras submetidas à tração uniaxial difere entre os 3 grupos de composição química. Observa-se que tanto os valores de Limite de escoamento (LE) como de Limite de resistência à tração (LRT) tendem a aumentar com o aumento do teor de impurezas na amostra. $O$ alongamento dos três grupos é aproximadamente igual. Observa-se também uma grande variação nos resultados obtidos para o Grupo 2 de amostras, refletido em valores altos de desvio-padrão.

Tabela 2. Propriedades mecânicas extraídas dos ensaios de tração

\begin{tabular}{lccc}
\hline & $\begin{array}{c}\text { Limite de } \\
\text { escoamento a 0,2\% } \\
(\mathrm{MPa})\end{array}$ & $\begin{array}{c}\text { Limite de resistência } \\
\text { (MPa) }\end{array}$ & Alongamento (\%) \\
\hline Grupo 1 & $46 \pm 2$ & $231,4 \pm 0,3$ & $40,5 \pm 0,3$ \\
Grupo 2 & $53 \pm 5$ & $243 \pm 10$ & $43 \pm 3$ \\
Grupo 3 & $81 \pm 3$ & $267 \pm 5$ & $42,1 \pm 0,6$ \\
\hline
\end{tabular}

A microestrutura resultante do recozimento também difere entre os 3 grupos de amostras. Como se observa na Figura 2, todas as amostras apresentam recristalização completa, porém a homogeneidade de tamanho de grão resultante variou com o teor de pureza da liga.

\footnotetext{
* Contribuição técnica ao 69ำ Congresso Anual da ABM - Internacional e ao 14ํㅡㄹ ENEMET - Encontro Nacional de Estudantes de Engenharia Metalúrgica, de Materiais e de Minas, 21 a 25 de julho de 2014, São Paulo, SP, Brasil.
} 

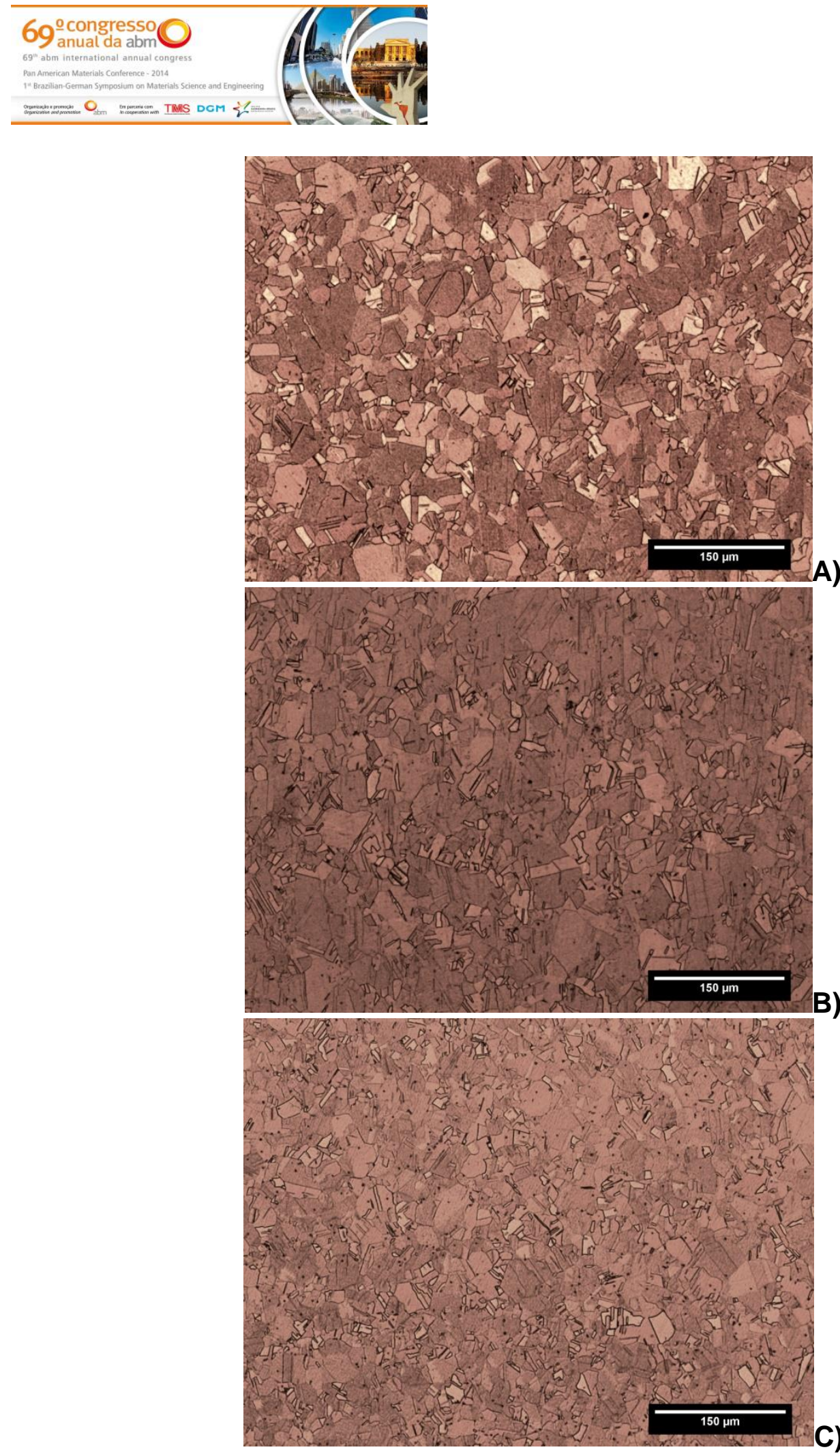

Figura 2. Micrografias típicas de cada grupo de amostra. A) Grupo 1; B) Grupo 2; e C) Grupo 3

Nota-se que as amostras do Grupo 2 (Figura 2.B), com pureza intermediária entre os grupos, apresentou a maior heterogeneidade de tamanho de grão. O Grupo 1 de amostras (Figura 2.A), com maior pureza, apresentou grãos maiores e alguma heterogeidade, enquanto o Grupo 3 (Figura 2.C), o de menor pureza, apresentou homogeneidade microestrutural, sendo o grupo com menor tamanho de grão entre as três.

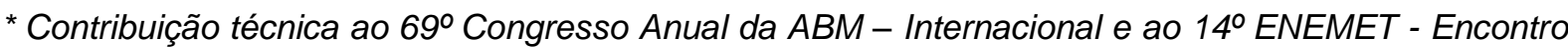
Nacional de Estudantes de Engenharia Metalúrgica, de Materiais e de Minas, 21 a 25 de julho de 2014, São Paulo, SP, Brasil.
} 


\section{DISCUSSÂO}

A partir dos resultados apresentados, constata-se que o comportamento mecânico das amostras variou significativamente para valores maiores com o somatório de impurezas presente nas mesmas, principalmente em termos de limite de escoamento e limite de resistência à tração. Quando cruzadas propriedades mecânicas e microestrutura das amostras, observa-se que essa variação com o teor de impurezas pode ser entendida, em primeira análise, em termos de endurecimento por solução sólida e endurecimento por redução do tamanho de grão.

O aumento do teor de impurezas de 184 ppm a 319 ppm parece causar um aumento na temperatura de recristalização, expressado na análise metalográfica pela diminuição do tamanho de grão à medida em que o teor de impurezas cresce. Estes resultados para o cobre fosforoso parecem confirmar a literatura para cobre eletrolítico [2-5].

Os resultados obtidos para o alongamento das amostras, no entanto, não são conclusivos a respeito da existência de uma influência do teor de impurezas. Segundo a escala proposta por Chmielarz e colaboradores [1], as principais impurezas presentes nas amostras deste estudo podem ser incluídas no grupo crítico $(\mathrm{Sn})$ e intermediário ( $\mathrm{Ni}, \mathrm{Fe}$ e $\mathrm{Zn}$ ) de influência sobre o alongamento de vergalhões medido por $A R$ Test. Os resultados, no entanto, não devem ser entendidos como divergentes, uma vez que se entendam as condições experimentais. Nos resultados apresentados por aqueles autores, a variação no alongamento total das amostras pode ser motivado por recristalização incompleta, devido à baixa temperatura e tempo de recozimento, o que não ocorreu nesse estudo. No entanto, aqueles autores não apresentaram análises metalográficas, o que impossibilita uma discussão mais conclusiva.

Deve-se também analisar a grande variabilidade das propriedades mecânicas das amostras do Grupo 2, com teor intermediário de impurezas. O desvio-padrão dos resultados, muito maior que para os demais grupos, pode ser explicado em termos da grande heterogeneidade microestrutural. Pode-se supor que a origem dessa heterogeneidade está relacionada a flutuações de composição química pela amostra, resultando em regiões puras e regiões mais impuras, em que a temperatura de recristalização e a oposição ao crescimento de grão variam. Ainda que flutuações possam ocorrer nas amostras dos grupos 1 e 3 , pode-se supor que 0 teor de impurezas mantém-se localmente abaixo (grupo 1) ou acima (grupo 3), de um limiar de influência sobre a temperatura de recristalização.

\section{CONCLUSÃO}

O teor de impurezas no cobre fosforoso (C12200) influencia a temperatura de recristalização do material, o que irá resultar, para temperatura e tempo fixos de recozimento, em variação de microestrutura e propriedades mecânicas.

Os resultados obtidos para o cobre fosforoso parecem confirmar a tendência apresentada na literatura para o cobre eletrolítico em termos de aumento de temperatura de recristalização do material.

Novos estudos sobre a evolução da microestrutura do material durante 0 recozimento são necessários para determinação das causas da heterogeneidade microestrutural e de propriedades mecânicas das amostras do Grupo 2 (total de 277 ppm de impurezas).

\footnotetext{
* Contribuição técnica ao $69^{\circ}$ Congresso Anual da ABM - Internacional e ao 14ํㅡㄹ ENEMET - Encontro Nacional de Estudantes de Engenharia Metalúrgica, de Materiais e de Minas, 21 a 25 de julho de 2014, São Paulo, SP, Brasil.
} 


\section{REFERÊNCIAS}

1 Chmielarz A, Baranek W, Krawiec G, Kopyto D. $7^{\text {th }}$ international copper conference; 2010 June 6-10; Hamburg, Germany. Proceedings of Copper 2010. ClausthalZellerfeld, (Niedersachsen): GDMB, 2010. 3023 p.

2 Silva BP, Giraldo CAS, Tschiptschin AP, Goldenstein, H. 14ํㅡㄹ Encontro nacional de tratamento de minérios e metalurgia extrativa; 2011 Outubro 16-19; Salvador, Brasil. Anais. Salvador, BA: UFBA, 2011.

3 Pitt CH, Tayler PL, Van Wagoner, DL. The effect of small amounts of Ag, Se, and Sn on the recrystallization of copper. Metallurgical Transaction A.1979, 10: 809-14.

4 Jakani S, Baudin T, de Novion C-H, Mathon M-H. Effect of impurities on the recrystallization texture in commercially pure copper-ETP wires. Materials Science and Engineering A. 2007, 456: 261-9.

5 Coutsouradis D, Diderrich E, Smets J, Crocq G, Pauwels L. Effects of trace amounts of impurities on the recrystallization behavior of high-purity, tough pitch copper.

Metallurgical Reports CRM. 1974, 39: 73

\footnotetext{
* Contribuição técnica ao $69^{\circ}$ Congresso Anual da ABM - Internacional e ao 14ํㅡㄹ ENEMET - Encontro Nacional de Estudantes de Engenharia Metalúrgica, de Materiais e de Minas, 21 a 25 de julho de 2014, São Paulo, SP, Brasil.
} 